\title{
Robot-Assisted Laparoscopic Resection of Large-Volume Pheochromocytoma through the Surface of the Kidney
}

\author{
Tao Ma, Zhenyu Cui*, Shichao Song, Yong Suo, Wenzeng Yang, Jingyang Guo \\ Department of Urology, Affiliated Hospital of Hebei University, Baoding, China \\ Email: matao197783@sina.com, *123109306@qq.com
}

How to cite this paper: Ma, T., Cui, Z.Y., Song, S.C., Suo, Y., Yang, W.Z. and Guo, J.Y. (2021) Robot-Assisted Laparoscopic Resection of Large-Volume Pheochromocytoma through the Surface of the Kidney. Surgical Science, 12, 391-398.

https://doi.org/10.4236/ss.2021.1212041

Received: August 18, 2021

Accepted: December 7, 2021

Published: December 10, 2021

Copyright $\odot 2021$ by author(s) and Scientific Research Publishing Inc. This work is licensed under the Creative Commons Attribution International License (CC BY 4.0).

http://creativecommons.org/licenses/by/4.0/

(c) (i) Open Access

\begin{abstract}
Objective: To investigate the clinical application of robot-assisted laparoscopic surgery on the treatment of large-volume pheochromocytoma. Methods: A retrospective analysis of 31 patients with pheochromocytoma disease admitted to the Department of Urology, Affiliated Hospital of Hebei University from May 2020 to August 2021. According to clinical data, the tumor volume was $62.4 \pm 29.5 \mathrm{~mm}$, including 16 males and 15 females, aged ( $35.6 \pm 16.1$ ) years old; 17 cases on the left side and 14 cases on the right side. All patients underwent anatomical resection through the renal superficial approach, and underwent pheochromocytoma resection through the retroperitoneal approach. The operation time, blood loss, postoperative complications, retention time of drainage tube and postoperative follow-up were observed. Results: All the 31 operations were successful, and there were no obvious postoperative complications. The average operation time, blood loss, and drainage tube retention time were $71 \mathrm{~min}, 80 \mathrm{~mL}$, and $(1.2 \pm$ 0.6) days, respectively. Postoperative pathology was confirmed to be pheochromocytoma. There was no tumor recurrence in the follow-up for 1 - 15 months. Conclusion: Robot-assisted laparoscopic anatomical resection of large-volume pheochromocytoma through the renal surface of the abdominal cavity can provide a larger operating space during the operation, with clear anatomical landmarks of "lipocrasis", less bleeding, less trauma, intraoperative and postoperative. The advantages of fewer complications, procedural operation, and shorter learning curve can be promoted in clinical practice.
\end{abstract}

\section{Keywords}

Surface of the Kidney, Robotic Surgery, Pheochromocytoma 


\section{Background}

Pheochromocytoma is a common adrenal gland disease in urology. Large tumor size and obesity will increase the difficulty of surgery. Hosam [1] believed that minimally invasive surgery is challenging for obese patients, and pointed out that there are few recent studies against robot-assisted adrenal surgery, especially in obese patients. He compared the surgical effect between robotic laparoscopic and traditional laparoscopic adrenal surgery, and concluded that robotic-assisted adrenal surgery is safe in obese patients. Compared with traditional laparoscopic surgery, it can improve postoperative results in certain aspects, such as reducing bleeding, and shorten the length of hospital stay. Bruhn [2] pointed out in the review that robot-assisted has unique advantages in visualization and anatomy, especially in challenging cases, such as large volume and adjacent to important blood vessels, etc. The therapeutic effects of traditional laparoscopy and robot laparoscopy are comparable. Robotic laparoscopy has the advantages of enhanced visualization and a faster learning curve. It can speed up dissection during surgery and increase the utilization of minimally invasive techniques. Through clinical practice, we have used robot-assisted retroperitoneal laparoscopic surgery through the kidney surface to treat large-volume pheochromocytomas and achieved satisfactory results. The concept of programmed precision anatomy was applied to the treatment of 31 cases of large-volume pheochromocytoma admitted from May 2020 to August 2021. The clinical effects, surgical points and experience are now introduced.

\section{Methods}

1) General data inclusion criteria

a) 31 cases of large-volume pheochromocytoma admitted between May 2020 and August 2021, with tumors were unilateral, localized adrenal lesion and radiographic largest axial tumour diameter $\geq 6 \mathrm{~cm}$; clinical symptoms, imaging examinations and laboratory tests of adrenal hormones supported the diagnosis of pheochromocytoma in the patients included in this study. The American Association of Anesthesiologists (ASA) classification was I-IV. b) Application of retroperitoneal robot-assisted retroperitoneal laparoscopic surgery. Exclusion criteria: a) Patients with bilateralpheochromocytoma or pheochromocytoma located outside the adrenal area were excluded; b) severe heart, brain, lung, liver and kidney dysfunction and severe coagulopathy; c) previous history of retroperitoneal surgery or infection on the affected side. A retrospective analysis showed that the tumor diameter was $62.4 \pm 29.5 \mathrm{~mm}$, including 16 males and 15 females, aged (35.6 \pm 16.1 ) years old; 17 cases on the left side and 14 cases on the right side. All patients completed B-ultrasound, thin-slice CT scan of the adrenal glands and other related examinations before surgery.

2) Surgical procedure

a) After general anesthesia, the posterior abdominal space is established. The robot assists the posterior abdominal cavity operation. After connecting the de- 
bugging equipment, the extraperitoneal fat is removed, and the lateral cone fascia is cut near the peritoneal reflex to enlarge the posterior abdominal cavity. $b$ ) After the abdominal cavity is established and enlarged, cut the perrenal fat on the side close to the peritoneum to find the surface of the renal parenchyma, which is an avascular layer. Expand along this level to properly free the surface of the kidney. The free range is determined according to the size of the tumor. The kidney shifts backward and downward, and the upper pole is separated from the tumor, fully revealing the adrenal area. At this time, the perrenal fat, adrenal fat, and the tumor is attached to the peritoneum and suspended above the kidney. Tumors and fat around the adrenal glands and perrenal fat will have obvious oval fissure-like structures. The larger the tumor, the fissure will become more obvious due to the expansion effect. Along this fissure, the pheochromocytoma peripheral fat and the perrenal fat will be separated, and the ventral surface will reach the peritoneum. The dorsal side reaches the psoas major muscle, and after these two layers expand and merge, only the base of the tumor remains. c) If the normal adrenal tissue remains very little or illegible, find the central vein just above the lower pole of the tumor and expose it. The Hemolock clip clamps the central vein and completely removes the adrenal glands and tumor. If surgery to retain part of the adrenal gland is feasible, place it above the central vein The Hemolock clip clamps the transected adrenal gland. Finally, the base of the tumor is cut off.

3) Observation and evaluation indicators

Operation time, blood loss, postoperative complications, drainage tube retention time and postoperative follow-up.

\section{Results}

The patients' baseline data are presented in Table 1. All 31 cases of pheochromocytoma were successfully operated without obvious postoperative complications.

Table 1. Preoperative profiles of patients in this study.

\begin{tabular}{cc} 
Variable & $\mathrm{N}=32$ \\
Demographic characteristics & \\
Age (years) & $35.6 \pm 16.1$ \\
Gender & \\
Female & 15 \\
Male & 16 \\
BMI (kg/m $\left.{ }^{2}\right)$ & $24.93 \pm 5.65$ \\
Tumor characteristics & \\
Laterality & 17 \\
Left & 14 \\
Right & $62.4 \pm 29.5$ \\
Diameter of tumor & \\
\hline
\end{tabular}


The average operation time, blood loss, drainage tube retention time and postoperative hospital stay were $71 \mathrm{~min}, 80 \mathrm{~mL}, 1.2 \pm 0.6$ days and $4.2 \pm 0.8$ days, respectively. Thirty-one patients were followed up for 1 to 15 months after operation, and no tumor recurrence was found. Perioperative and prognosis data of patients are in Table 2.

\section{Discussion}

With the renewal of equipment and the advancement of surgical technology, the application of robot-assisted surgery in the field of surgery has been accepted. This emerging technology can be safely and widely used in surgical operations. Due to the ergonomic design, the three-dimensional view of the operating area, and the precise operation of the robotic arm, robotics technology has advantages in many aspects. In recent years, robot-assisted adrenal surgery has been adopted in many medical centers [3]. Especially to deal with more complicated cases. Although the guidelines recommend open surgery for large-volume pheochromocytomas, many studies currently underway show that minimally invasive surgery is feasible for large tumors over $6 \mathrm{~cm}$, and the results of intraoperative and postoperative complications are very similar. There is no difference in open surgery [4]. At the current stage, traditional laparoscopic adrenal surgery is still the "standard procedure". Fiori [5] pointed out that laparoscopy is the first choice for small and medium-sized benign adrenal tumors, but robotic adrenal surgery has become more and more popular recently, and its surgical effect It is completely comparable to laparoscopy, especially when dealing with large-volume tumors. Morelli [6] comparative studies have shown the potential benefits of robotic surgery compared with traditional laparoscopic surgery, especially for patients with a tumor $\geq 6 \mathrm{~cm}, \mathrm{BMI} \geq 30 \mathrm{~kg} / \mathrm{m}^{2}$, and a history of surgery in the surgical area. Many studies have provided a basis for robotic laparoscopic surgery to treat large-volume tumors, showing its effectiveness and safety. Robotic surgery can enhance the precise operation of the operation. The

Table 2. Perioperative and prognosis data of patients in this study.

$\begin{array}{cc}\text { Variable } & \mathrm{n}=32 \\ \text { Intraoperative parameters } & 71 \\ \text { Operating time (min) } & 80 \\ \text { Intraoperative bleeding }(\mathrm{ml}) & \\ \text { Postoperative data } & 1.2 \pm 0.6 \\ \text { Time to removal of drainage (days) } & 4.2 \pm 0.8 \\ \text { Postoperative hospitalization days } & 1-15 \\ \text { Follow-up data } & 0 \\ \text { Duration of follow-up (month) } & \\ \text { Recurrence rate (\%) } & \end{array}$


domestic Changhai Hospital adopts robot-assisted adrenal surgery. The main blood vessels of the adrenal gland are clamped and the tumor is accurately removed. The average warm ischemia time is 12 minutes. The average blood loss was $20 \mathrm{~mL}$. No postoperative complications, a median follow-up of 12 months after the operation, no recurrence of the disease was found [7]. Cochetti [8] reported that a giant adrenal medullary lipoma underwent robotic laparoscopic surgery. The patient's examination revealed that the size of the right adrenal gland tumor was $16 \times 13 \mathrm{~cm}$. Robotic laparoscopic tumor resection was performed and part of the normal gland tissue was preserved. The operation time and estimated blood loss were 205 minutes and $100 \mathrm{ml}$, respectively. No intraoperative or postoperative complications occurred. Robotic surgery has obvious advantages in some aspects, but it cannot be ignored that there are also certain limitations at the current stage, such as popularity and economic reasons. Ruhle [9] pointed out that although robotic surgery provides surgeons with good ergonomics and speeds up patient recovery, the main disadvantage of robotic systems is their high cost. As new robotic-assisted surgery equipment reduces costs and accelerates innovation, robotic surgery may expand significantly.

In recent years, minimally invasive surgery for the adrenal gland has been developing steadily. As the use of robotic surgery increases, robotic adrenal surgery, especially retroperitoneal laparoscopic surgery, has been regarded as a good choice by many large medical centers. Inversini [10] reviewed the latest literature from May 2010 to November 2019, and pointed out that whether robotic surgery is truly superior to traditional minimally invasive methods is still controversial. Robotic surgery is safer due to reduced hospital stay, reduced blood loss and equivalent complication rate, but despite these advantages, the operating time and total cost of robotic surgery are still higher than traditional laparoscopy. The stratification of patients eligible for robotic surgery should be further optimized. Compared with trans-abdominal surgery, retroperitoneal surgery has its own advantages and disadvantages. For posterior abdominal surgery, many scholars believe that the operating space is relatively narrow. There is no clear anatomical landmark, but it can avoid entering the abdominal cavity, reducing the risk of abdominal organ damage and intestinal obstruction. Transperitoneal surgery can obtain a larger space and many anatomical landmarks, but it inevitably needs to enter the abdominal cavity and increase the interference of internal organs in the abdominal cavity. If you undergo retroperitoneal surgery, you can solve the disadvantages of narrow space. In addition, it is found that clear anatomical landmarks can be used as guidelines to maximize strengths and avoid weaknesses, and it is more suitable for clinical use. Resection of large-volume pheochromocytoma through the retroperitoneal cavity requires two problems to be resolved. First, the operation space should be large enough. Second, find a clear "lighthouse" anatomical landmark. We will solve the above problems from the following details during the operation. First, the process of establishing the posterior abdominal space. The expansion of the posterior ab- 
dominal cavity should be sufficient, and the balloon should be filled with 800 $1000 \mathrm{ml}$. Secondly, after the mechanical arm is inserted, the lateral cone fascia must be fully incised from top to bottom when opening the lateral cone fascia. In the process, special attention must be paid to avoid damaging the peritoneum, otherwise the effective space of the posterior abdominal cavity will be lost. After opening the lateral cone fascia, cut the perrenal fat sac for the first time and free it along the surface of the kidney. At this time, the free range is determined by the size of the tumor. If the volume of the tumor is relatively small, the free area of the kidney can be appropriately reduced. If the volume of the tumor is large, the free range needs to be increased. If necessary, the entire kidney can be freed to increase the activity of the kidney and gain more space. After the kidney is fully free, the kidney shifts backward and downward, and the kidney is separated from the tumor area. At this time, the posterior abdominal tumor will be fully exposed. At this time, carefully identify the adipose tissue attached to the posterior adrenal abdominal mass. There will be a more obvious fissure between the attached adipose tissue and the peripheral adipose tissue, which we call "fatty fracture". The abdominal mass tissue after freeing along this fat fissure is completely excised. There are several advantages to freeing the tumor along the fat fissure. First, if the tumor capsule is thin and free along the fat fissure layer, it can reduce the chance of damaging the tumor capsule, keep the tumor intact, and avoid causing the tumor tissue to overflow. Second, pheochromocytoma has a rich blood supply and is rich in swollen veins. Operating along the level of fat fissures can reduce the bleeding of the tumor to a certain extent. Third, the middle of the fat fissure is an area of depleted blood vessels. Freeing along this fissure can avoid blood oozing and maintain a clear vision. Fourth, this fissure is a ubiquitous natural fissure, regardless of the size of the patient who is thin or obese, this fissure can be found after identification, and it can be identified as a clear anatomical landmark.

In summary, we have the following experience. Firstly, robot-assisted laparoscopic surgery for large-volume pheochromocytoma has advantages in certain aspects and can be a clinical choice. Secondly, after the posterior abdominal cavity is freed from the kidney surface, a larger operation space can be obtained, which creates conditions for the treatment of large-volume tumors. Thirdly, adrenal surgery following the "fatty split" sign has the advantages of simplified procedures, clear anatomical signs, low probability of secondary injury, and short learning curve. Finally, during the operation, the fat fissure along the adrenal gland can be freed, which can reduce adrenal harassment and avoid fluctuations in blood pressure and heart rate during the operation. During the operation, it can reduce the risk of tumor damage and bleeding.

\section{Conclusion}

Robot-assisted laparoscopic anatomical resection of large-volume pheochromocytoma through the renal surface of the abdominal cavity can provide a larger 
operating space during the operation, with clear anatomical landmarks of "lipocrasis", less bleeding, less trauma, intraoperative and postoperative. The advantages of fewer complications, procedural operation, and shorter learning curve can be promoted in clinical practice.

\section{Limitations}

The disadvantage of this study is that there is no control group. It is planned to compare data with other surgical methods in the near future, and conduct deeper anatomical observation studies to provide stronger evidence for its clinical application.

\section{Founding}

Science and Technology Plan Project of Baoding City, Hebei Province Project Number: 2041ZF164.

\section{Conflicts of Interest}

The authors declare no conflicts of interest regarding the publication of this paper.

\section{References}

[1] Shalaby, H., Abdelgawad, M., Omar, Md.M., et al. (2021) Robotic and Laparoscopic Approaches for Adrenal Surgery in Obese Patients. The American Surgeon, 87, 588-594. https://doi.org/10.1177/0003134820951498

[2] Bruhn, A.M., Hyams, E.S., Stifelman, M.D. and Stifelman. (2010) Laparoscopic and Robotic Assisted Adrenal Surgery. Minerva Urology and Nephrology, 62, 305-318.

[3] Makay, O., Erol, V. and Ozdemir, M. (2019) Robotic Adrenalectomy. Gland Surgery, 8, S10-S16. https://doi.org/10.21037/gs.2019.01.09

[4] Sahbaz, N.A., Dural, A.C., Akarsu, C., et al. (2020) Transperitoneal Laparoscopic Surgery in Large Adrenal Masses. Wideochir Inne Tech Maloinwazyjne, 15 106-111. https://doi.org/10.5114/wiitm.2019.85177

[5] Fiori, C., Checcucci, E., Amparore, D., et al. (2020) Adrenal Tumours: Open Surgery versus Minimally Invasive Surgery. Current Opinion in Oncology, 32, 27-34. https://doi.org/10.1097/CCO.0000000000000594

[6] Morelli, L., Tartaglia, D., Bronzoni, J., et al. (2016) Robotic Assisted versus Pure Laparoscopic Surgery of the Adrenal Glands: A Case-Control Study Comparing Surgical Techniques. Langenbeck's Archives of Surgery, 401, 999-1006. https://doi.org/10.1007/s00423-016-1494-0

[7] Ye, C., Yang, Y., Guo, F., et al. (2020) Robotic Enucleation of Adrenal Masses: Technique and Outcomes. World Journal of Urology, 38, 853-858. https://doi.org/10.1007/s00345-019-02868-7

[8] Cochetti, G., Paladini, A., Boni, A., et al. (2019) Robotic Treatment of Giant Adrenal Myelolipoma: A Case Report and Review of the Literature. Molecular and Clinical Oncology, 10, 492-496. https://doi.org/10.3892/mco.2019.1823

[9] Ruhle, B.C., Bryan, A.F. and Grogan, R.H. (2019) Robot-Assisted Endocrine Surgery: Indications and Drawbacks. Journal of Laparoendoscopic \& Advanced Surgic- 
al Techniques, 29, 129-135. https://doi.org/10.1089/lap.2018.0308

[10] Inversini, D., Manfredini, L., Galli, F., et al. (2020) Risk Factors for Complications after Robotic Adrenalectomy: A Review. Gland Surgery, 9, 826-830.

https://doi.org/10.21037/gs.2020.04.10 